\title{
Thymoquinone dose-dependently attenuates myocardial injury induced by isoproterenol in rats via integrated modulation of oxidative stress, inflammation, apoptosis, autophagy and fibrosis
}

Mahmoud Mohamed Farag ( $\square$ mahmoudfarag2012@hotmail.com )

MRI: Alexandria University Medical Research Institute

Asmaa Ahmed Khalifa

Alexandria University Medical Research Institute

Wessam Fahmy El-Hadidy

Alexandria University Medical Research Institute

Radwa Mohamed Rashad

Alexandria University Medical Research Institute

\section{Research Article}

Keywords: Mycardial infarction, Thymoquinone, Oxidative stress, Inflammation-Fibrosis, Apoptosis, Autophagy

Posted Date: March 9th, 2021

DOI: https://doi.org/10.21203/rs.3.rs-169928/v1

License: (c) (i) This work is licensed under a Creative Commons Attribution 4.0 International License.

Read Full License 


\section{Abstract}

As rats develop myocardial infarction (MI) like lesions when injected with large doses of isoproterenol (ISO), this investigation was designed to evaluate the effects of low and high doses of thymoquinone (TQ) on ISO-induced myocardial injury in rats. Adult male rats were divided into control, TQ20 (20 $\mathrm{mg} / \mathrm{kg} /$ day), TQ50 (50 mg/kg/day), and ISO, TQ20 + ISO and TQ50 + ISO groups. In these rats, biochemical, immunobiochemical and histopathological studies were carried out to evaluate myocardial oxidative stress, inflammation, apoptosis, fibrosis and autophagy and serum cardiac biomarkers. The results showed that TQ pretreatment in ISO-administered rats produced a dose-dependent significant reduction of the myocardial infarct size, markedly reduced the ISO-induced elevation in serum cardiac markers and demonstrated several other important findings related to the cardioprotective efficacy of TQ. First, this study is the first reported research work showing that TQ treatment could increase the myocardial reduced glutathione baseline level, adding an indirect antioxidant effect to its known direct free radical scavenging effect. Second, pretreatment with TQ significantly reduced the markers of myocardial oxidative stress, inflammation, fibrosis and apoptosis. Third, TQ acted as an autophagy enhancer ameliorating myocardial cell damage and dysfunction. Thus, the changes associated with ISOinduced myocardial injury were ameliorated with TQ pretreatment. Additionally, the extent of observed improvement was significantly greater with the high TQ dose than with the low dose use. These findings raise the possibility that TQ may serve as a promising prophylactic cardioprotective therapy for patients who are at risk of developing myocardial injury as in cases of MI.

\section{Introduction}

Myocardial infarction (MI) continues to be a major public health problem and one of the leading causes of morbidity and mortality in both developed and non-developed countries (Moran et al. 2014; Nascimento et al. 2019). This condition occurs due to the imbalance between coronary blood supply and myocardial demands. In the ischemic tissues, the generated oxygen free radicals are implicated in oxidative chain reactions, which damage the cell membrane and subcellular structures containing phospholipids, proteins and DNA (Halliwell 1991). Subsequently, these reactions lead to functional, structural and metabolic alterations in the heart (He and Zuo 2015).

Animals develop heart infarct like lesions when injected with large doses of isoproterenol (ISO), a potent synthetic catecholamine, which induces myocardial hypertrophy, myocyte damage and contractile dysfunction (Rona 1985). These lesions are morphologically similar to those of "coagulative myocytolysis", a type of necrosis which characterizes MI in human (Milei et al. 1978; Leone 2017). Experimental studies on ISO-induced cardiotoxicity have clearly demonstrated the involvement of oxidative stress in the pathogenesis of this condition and the positive correlation of free radicals with endothelial injury and cardiovascular disease progression (Saravanan and Parkash 2004; Wang et al. 2016). 
Therapeutic approaches that could minimize excess free radical production in the ischemic myocardium or improve impaired endogenous antioxidant defense mechanisms have been of great interest to protect the heart against the detrimental effects of acute tissue injury (Somaiya et al. 2015). The herbal medicine has attracted much attention and there has been an upsurge of interest to explore the cardioprotective potential of natural products as alternatives to chemical drugs (Upaganlawar et al. 2011;Caricati-Neto et al. 2019). One of these compounds is thymoquinone (TQ, 2-isopropyl-5-methylbenzo-1, 4-quinone) which has been a subject of interest in pharmacological studies. It is the main active ingredient derived from Nigella sativa seeds, commonly used as a food supplement and in folk medicine (Ahmad et al. 2013). Thymoquinone reduces the generation of reactive oxygen species (ROS) and suppresses oxidative stressinduced oxidative damage in several tissues as in cyclosporine A-induced nephrotoxicity (Farag et al. 2015), acetaminophen-induced hepatotoxicity (Awad et al. 2016) and doxorubicin-induced cardiotoxicity (Pei et al. 2018). Thymoquinone is regarded by many investigators as a potent antioxidant with additional anti-inflammatory properties that may expand its therapeutic potential especially in cardiovascular diseases (Shabana et al. 2013).

The cardioprotective activity of TQ is complex and not completely clarified. Hence, the aim of the present study was to evaluate the potential cardioprotective effect of low and high doses of TQ against ISOinduced acute myocardial injury in rats. The current investigation focused on the effects of TQ on the main pathological events including oxidative stress, inflammation, apoptosis, fibrosis and autophagy involved in the initiation and progression of acute myocardial tissue injury.

\section{Materials And Methods}

\section{Drugs and chemicals}

Thymoquinone and isoproterenol (ISO) powders were obtained from Sigma Aldrich, Germany. Dimethyl sulfoxide (DMSO, prepared as 5\% solution) was obtained from Arabic Laboratory Equipment Co., Cairo, Egypt. All the used chemicals were of a high analytical grade and were purchased from Sigma Chemical Co. Cairo, Egypt.

\section{Animals and experimental protocol}

Ninety two male albino rats weighing 150-200 g were used in this study. Rats were housed in cages under good sanitary conditions and normal humidity $(60 \%)$, with free access to food and water during the experimental period. The rats were maintained in the animal research facility under standard conditions of temperature $20-22^{\circ} \mathrm{C}$ with a 12 -h light-dark cycle.

In this study, the rats were randomly assigned into the following six experimental groups (14-16 rats per each group):

Group 1: Normal control group received 5\% DMSO (1 ml, orally daily) for 21 days and 
received normal saline $(0.5 \mathrm{ml} / \mathrm{kg}$, s.c. $)$ on the $20^{\text {th }}$ and $21^{\text {st }}$ days at a $24 \mathrm{~h}$ interval.

Group 2: Thymoquinone (TQ 20) control group received TQ at a dose of $20 \mathrm{mg} / \mathrm{kg} / \mathrm{day}$

(Ojha et al. 2015) orally dissolved in $5 \%$ DMSO, for 21 days. On the $20^{\text {th }}$ and $21^{\text {st }}$ days, normal saline was administered $(0.5 \mathrm{ml} / \mathrm{kg}$, s.c., at a $24 \mathrm{~h}$ interval).

Group 3: Thymoquinone (TQ 50) control group received TQ at a dose of $50 \mathrm{mg} / \mathrm{kg} / \mathrm{day}$ (Pei et al. 2018) dissolved in 5\% DMSO, orally for 21 days. On the $20^{\text {th }}$ and $21^{\text {st }}$ days, normal saline was administered as in group 1.

Group 4: Isoproterenol (ISO) group received 5\% DMSO (1 ml, orally daily) for 21 days and received ISO at a dose of $85 \mathrm{mg} / \mathrm{kg}$ (Ojha et al. 2015) dissolved in $0.5 \mathrm{ml}$ saline, s.c., on the $20^{\text {th }}$ and $21^{\text {st }}$ days (at an interval of $24 \mathrm{~h}$ ).

Group 5: Thymoquinone 20 + Isoproterenol (TQ 20+ISO) group received TQ 20 mg/kg/day (dissolved in $1 \mathrm{ml} 5 \%$ DMSO, orally) for 21 days along with concurrent administration of ISO on the $20^{\text {th }}$ and $21^{\text {st }}$ days as in group 4 .

Group 6: Thymoquinone $50+$ Isoproterenol (TQ $50+1 \mathrm{SO}$ ): the rats of this group received TQ $50 \mathrm{mg} / \mathrm{kg} /$ day, (dissolved in $1 \mathrm{ml} 5 \%$ DMSO, orally) for 21 days along with concurrent administration of ISO the $20^{\text {th }}$ and $21^{\text {st }}$ days as in group 4 .

Twenty four hours after the second s.c. injection of ISO (or normal saline), the rats were sacrificed. Blood was collected from the abdominal aorta, allowed to clot for $30 \mathrm{~min}$ at room temperature and serum was separated and stored at $-20^{\circ} \mathrm{C}$ until used for measuring serum cardiac markers. The hearts of each group were excised immediately, washed in ice- cold phosphate buffer saline and randomly divided into two subgroups (7-8 hearts per group). The first subgroup was used for the measurement of myocardial infarct size. The second subgroup was used to prepare 10\% homogenates in phosphate buffer $(\mathrm{pH} 7.4)$ and kept at $-80^{\circ} \mathrm{C}$ until used for myocardial tissue biochemical assays.

\section{Myocardial infarct area measurement}

The direct triphenyl tetrazolium chloride (TTC) assay was used to determine the myocardial infarct area as described by Lie et al. (1975). Briefly, the hearts were washed with ice cold saline and quickly semifrozen at $-20^{\circ} \mathrm{C}$ for $40 \mathrm{~min}$. Each heart was transversely cut across the left ventricle, sections of 2-3 mm 
thickness were incubated in $1 \%$ TTC solution prepared in phosphate buffer $\left(\mathrm{pH} \mathrm{7.4)}\right.$ at $37^{\circ} \mathrm{C}$ for $30 \mathrm{~min}$ and then fixed with $10 \%(\mathrm{~V} / \mathrm{V})$ neutral buffered formalin solution. Then the slices were put between two slides. The non-ischemic myocardium and viable ischemic myocardium were stained red, while the infarcted myocardium appeared pale gray or white. The slices were photographed on white background using digital camera with a ring flash at high resolution. The infarcted area, expressed as percentage of the area left ventricle, in each image was analyzed on Intel ${ }^{\circledR}$ Core $i 5^{\circledR}$-based computer using the Image $\mathrm{J}^{\circledR}$ software, Java-based image processing program (National Institutes of Health and the Laboratory for Optical and Computational Instrumentation, USA).

\section{Assays of cardiac markers enzymes in serum}

The activity of serum aspartate aminotransferase (AST) was determined colorimetrically using a Tecno diagnostic kit (Tecno diagnostic Co., USA). Also, the levels of creatine phosphokinase-myocardial band (CK-MB), lactate dehydrogenase (LDH) and cardiac troponin I (c Tn I) were measured spectrophotometrically in serum using rat enzyme-linked immunosorbent assay (ELISA) kits (My Biosource Co., USA).

\section{Assays in heart homogenate}

- Oxidative stress markers

The reduced glutathione (GSH) content was determined by a method based on the reduction of $5,5^{\prime}$ dithiobis-2-nitrobenzoic acid with GSH as described previously (Richardson and Murphy 1975) and lipid peroxidation was estimated by the thiobarbituric acid reaction by measuring the malondialdehyde (MDA) level (Ohkawa et al. 1979). For superoxide dismutase (SOD) assay, the activity of the enzyme was determined by a method based on the inhibition of phenazine methosulfate-mediated reduction of nitroblue tetrazolium dye with SOD (Nishikimi et al. 1972).

- Determination of proinflammatory cytokines:

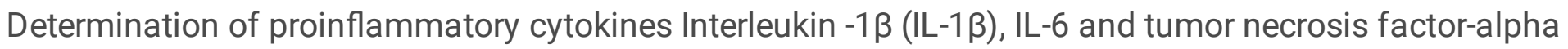
(TNF-a) levels in the heart homogenate was performed by using rat ELISA kits (My Biosource Co., USA).

- Measurement of caspase- 3 activity

Caspase-3 activity, as a marker of apoptosis, was measured colorimetrically in the heart homogenate using a rat Caspase-3 ELISA Assay kit (My Biosource Co., USA), following the instructions of the manufacturer.

- Determination of microtubule-associated protein light chain 3 (LC3) level

The LC3 western blotting (immunoblotting) assay method was used in this study to evaluate autophagy in the myocardial tissue (Klionsky et al., 2007). In brief, protein extraction was performed in 
radioimmunoprecipitation assay buffer, separated by sodium dodecyl sulfate polyacrylamide gel electrophoresis and transferred to a polyvinylidene fluoride membrane. This membrane was incubated with primary antibodies (anti-LC3 antibodies) at $4{ }^{\circ} \mathrm{C}$ overnight followed by incubation with horseradish peroxidase-conjugated secondary antibodies for one hour at room temperature. The bands were visualized using a chemiluminescence detection kit (Bio-Rad, USA) and image analysis software was used to read the band intensity against a control sample after normalization by $\beta$-actin (The procedure of this assay, including the types of the antibodies used and image analysis, is described in the Supplementary materials).

\section{Histopathological examination}

Immediately after excision, rat heart tissue from each group was fixed in $10 \%$ buffered neutral formalin solution and, thereafter, dehydrated in graded alcohol and embedded in paraffin wax. Four

micron-thick sections were cut and stained with either hematoxylin and eosin (to evaluate inflammatory and necrotic changes) or Masson's trichrome stain (to evaluate necrotic and fibrotic changes). The heart tissue sections were examined under light microscopy.

\section{Statistical analysis}

The data are presented as mean \pm SEM. SPSS statistical package (version 11.5 software) was used for statistical analysis. One-way analysis of variance (ANOVA) followed by post-hoc Tukey \s test were used to assess the significance of differences among the experimental groups. Pearson $\nabla \mathrm{s}$ test was used to examine the correlations between various parameters. Values of $\mathrm{P} \otimes 0.05$ were considered statistically significant.

\section{Results}

\section{Myocardial damage area results}

Images of transverse sections of heart from control and drug-treated rats are shown in Fig. 1a. In control rats, the viable myocardial tissue was stained brick red while in ISO-treated rats the necrotic infarcted myocardial tissue did not stain with TTC and was visible as an unstained area. Figure $1 \mathrm{~b}$ shows the cardiac infarction area measurements, expressed as percentage of the left ventricular area. In rats of the control, TQ20 and TQ50 groups, no evidence of myocardial injury was detected in their hearts. A significant improvement in the measures of the area of ISO-induced myocardial injury was observed in the rats treated with TQ before and concomitantly with ISO administration. The large percentage (39.9\%) of the myocardial injured area in the vehicle-pretreated ISO group was markedly reduced to $13.9 \%$ and $3.8 \%$ in the TQ20+ISO and TQ50+ISO groups, respectively $(P<0.05)$. The differences between the latter two groups were statistically significant suggesting that the protective effect of TQ was dose-dependent.

\section{Serum biochemical results}


As shown in Table 1, administration of ISO to the vehicle (DMSO)-treated rats, in the present study, significantly increased serum activities of AST, CK-MB and LDH by 307, 452 and 455\%, respectively, as compared to the corresponding control values. In rats pretreated with TQ at a dose of 20 or 50 $\mathrm{mg} / \mathrm{kg} /$ day, the ISO-induced increases in serum AST activity were reduced to 89 and $40 \%$, respectively, as compared to the control value and the LDH activity decreased to 144 and $73 \%$, respectively, as compared to the control value. Also, the increase in serum CK-MB activity was reduced to $110 \%$ of the control value in rats pretreated with the lower dose of TQ before ISO administration. In rats pretreated with the larger dose of TQ, serum CK-MB activity was normalized as no significant difference was detected in comparison to control values.

Troponin-I levels in serum were significantly increased $(P<0.05)$ in ISO-treated rats by $813 \%$ as compared to control rats (Table 1). In rats treated with the low dose of TQ before and concomitantly with ISO administration showed a significant decrease $(P<0.05)$ in the ISO-induced elevation in serum troponin-I level to 258 \% as compared to the control value. In rats treated with the larger dose of TQ before and concomitantly with ISO administration, the serum troponin-I levels were close to the corresponding values in control rats without a significant difference $(P>0.05)$.

\section{Cardiac tissue IL-1 $\beta$, IL-6 and TNF-a levels}

Figure 2 shows the effect of TQ on the cardiac tissue levels of the proinflammatory cytokines, IL-1 3 , IL-6 and TNF-a in ISO-treated rats. A marked increase in cardiac tissue IL-1 $\beta$, IL-6 and TNF-a levels was observed in ISO-treated control rats as compared to saline-treated control rats. Interestingly, TQ administration before and concomitantly with ISO treatment significantly reduced $(P<0.05)$ the ISOinduced elevations in cardiac tissue levels of these cytokines. As demonstrated in Fig. 2, the rats treated with the larger dose of TQ showed a greater decline in the cardiac tissue levels of the three cytokines as compared to the rats treated with the lower dose of TQ $(P<0.05)$.

\section{Cardiac tissue oxidant/antioxidant markers}

The myocardial lipid peroxidation marker, MDA content, was significantly increased by $628 \%$ in the ISO group as compared to the normal control group (Fig. 3). In rats treated with TQ at doses of 20 and 50 $\mathrm{mg} / \mathrm{kg} /$ day before and concomitantly with ISO administration, the observed increase in myocardial MDA content induced by ISO showed a significant dose-dependent reduction to $218 \%$ and $79 \%$, respectively, as compared to the normal control group $(P<0.05)$.

As shown in Fig. 3, in control rats treated with the high dose of TQ (50 mg/kg/day) for 21 days, the cardiac tissue GSH content was 29.24 \pm 0.88 (mean \pm SEM) pg/g tissue, whereas the vehicle (DMSO)treated control rats showed a significantly lower cardiac tissue GSH content $(24.14 \pm 1.28 \mathrm{pg} / \mathrm{g}$ tissue, $P<$ $0.05)$. A nonsignificant increase $(P>0.05)$ in this parameter was observed in the control group treated with the lower dose of TQ, as compared to the vehicle-treated control group. As demonstrated in Fig. 3, a significant fall by $83 \%$ in the cardiac tissue GSH content was observed in the ISO group, as compared to the normal control group $(P<0.05)$ and a significant dose-dependent attenuation of this fall was 
observed in rats treated with TQ at doses of 20 and $50 \mathrm{mg} / \mathrm{kg} /$ day before and concomitantly with ISO injection. In the latter two experimental groups, the decline in cardiac tissue GSH content was $49 \%$ and $18 \%$, respectively, as compared to the normal control group (Fig. 3).

Similarly, a marked fall in the cardiac tissue SOD activity was observed in ISO-challenged rats. This enzyme activity was reduced by $92 \%$ in the ISO group, as compared to the normal control group $(P<$ $0.05)$. This percentage of reduction in the cardiac tissue SOD activity was significantly attenuated $(P<$ 0.05 , Fig. 3) in a dose-dependent manner to $59 \%$ and $30 \%$ in the groups of rats treated with TQ at the low and high doses, respectively, before and concomitantly with ISO administration.

\section{Cardiac tissue caspase-3 level}

As shown in Fig. 4a, ISO administration to rats pre-and co-treated with the vehicle (DMSO) resulted in an increase of cardiac tissue caspase-3 level, a marker of cell apoptosis, by $756 \%$, as compared to normal control rats. Amelioration of this massive elevation was observed in rats pre- and co-treated with TQ as this percentage of the ISO-induced increase in caspase-3 level was significantly lowered $(P<0.05)$ to $224 \%$ and $77 \%$, as compared to the normal control group, in rats pre-and co-treated with TQ at doses 20 and $50 \mathrm{mg} / \mathrm{kg} / \mathrm{day}$, respectively. The difference between the latter two groups was statistically significant $(P<0.05)$.

\section{Cardiac tissue LC3 level}

To evaluate myocardial autophagy, an immunoblotting analysis to measure the cardiac tissue LC3 level was performed. As indicated in Fig. 4b, the basal cardiac tissue levels of LC3, an autophagic marker, were increased by $12 \%$ and $44 \%$ in the control groups treated with TQ at doses of 20 and $50 \mathrm{mg} / \mathrm{kg} / \mathrm{day}$, respectively, as compared to the vehicle (DMSO)-treated control group. The cardiac tissue LC3 levels in the rats treated with the larger dose of TQ was statistically significant, as compared to the corresponding values in both DMSO- and lower TQ dose-treated control groups $(P<0.05)$.

As shown in Fig. 4b, ISO administration to DMSO-pretreated rats resulted in a $84 \%$ reduction in the cardiac tissue LC3 level $(P<0.05)$. This percentage of reduction was significantly attenuated, in a dosedependent manner, to $40 \%$ and $20 \%$ in the groups treated with TQ at doses of 20 and $50 \mathrm{mg} / \mathrm{kg} / \mathrm{day}$, respectively, before and concomitantly with ISO administration.

\section{Correlation results}

As shown in Table 2, using the data of all experimental groups, there were highly significant positive correlations between each one of the measured serum cardiac biomarkers (AST, CK-MB, LDH and troponin-I) and the levels of proinflammatory cytokines (IL-1 $\beta$, IL- 6 and TNF- $\alpha$ ) in the myocardial tissue ( $P$ $<0.001)$. Also, the myocardial tissue MDA level was significantly and positively correlated with the serum biomarkers AST, CK-MB, LDH and troponin-I, $P<0.05$. Conversely, each one of these biomarkers was significantly and inversely correlated with the levels of both GSH content and SOD activity in the myocardial tissue $(P<0.001$, Table 2). Our correlation studies also demonstrated a significant positive 
correlation $(P<0.001)$ between the area of the injured myocardium and the tissue level of caspase-3, as a member of the cysteine- aspartic acid protease family that plays a vital role in cell apoptosis, whereas a significant negative correlation between the injured area and the myocardial tissue levels of LC3, as a marker of autophagy, was observed (Figs. 4 c \& d, respectively).

\section{Histopathological results}

- Histopathological findings with hematoxylin-eosin (H\&E) staining

Microscopic examination of heart tissue sections of control rats (Fig. 5a) showed a normal cardiac histoarchitechture with a well- balanced myofibrillar structure, elongated nuclei and regular crossstriations. No inflammatory cell infiltration was observed in the heart sections of control rats. Examination of sections of heart tissue from TQ (20 or $50 \mathrm{mg} / \mathrm{kg} /$ day) treated rats (Fig. $5 \mathrm{~b} \& \mathrm{c}$, respectively) depicted apparently normal heart architecture with no histopathological changes.

Cardiac tissue sections of ISO-treated rats (Fig.5d) exhibited several histopathological changes including myocardial cell separation, inflammatory mononuclear cell (lymphocytes and macrophages) infiltration in contact with necrotic myocytes, loss of transverse striations and interstitial edema along with vascular congestion and extravasation of red blood cells.

Thymoquinone ( 20 or $50 \mathrm{mg} / \mathrm{kg} /$ day) treatment before and concomitantly with ISO administration ameliorated the histopathological changes induced by ISO. In the TQ20+ ISO group, the cardiac sections showed slightly separated myocardial fibers, scattered few inflammatory cells with lessened patchy myocardial necrotic damage and edema (Fig.5e). These histopathological changes were very mild and apparently normal cardiac myocytes were observed in cardiac sections of rats treated with the larger dose of TQ (Fig. 5f).

- Histopathological findings with Masson's trichrome staining

As Masson's trichrome staining helps to detect fibrotic changes, it was used in the present study for the histopathological assessment of myocardial injury. Using this stain, the nuclei are stained in black, the cell cytoplasm is stained in red and collagen fibrils and fibers are stained in blue. As shown in Fig. 6, a scant amount of interstitial delicate collagen fibrils associated with normal cardiac histoarchitecture was observed on microscopic examination of slides of cardiac tissue from control rats treated with the vehicle (Fig. 6a) or TQ at a dose of 20 or $50 \mathrm{mg} / \mathrm{kg} /$ day (Fig. 6 b \& c, respectively).

Compared to the control group, the cardiac tissue sections from ISO-treated rats exhibited widespread thick and dense amount of collagen fibrils associated with necrotic myocytes in interstitial and perivascular areas (Fig. 6 d). However, the cardiac tissue sections from rats treated with TQ before and concomitantly with ISO administration showed less fibrous tissue deposition, few interstitial collagen fibrils and marked resemblance to the normal cardiac histoarchitecture especially in rats pretreated with the high dose of TQ (Fig. 6 f) as compared to the rats pretreated with the low dose (Fig. 6 e). 


\section{Discussion}

In this study, we used the rat model of ISO-induced cardiotoxicity that demonstrates an infarct-like myocardial lesion with major serum and myocardial tissue changes similar to those occurring in human acute MI disease (Milei et al. 1978; Ojha et al. 2012; Leone 2017). With myocardial injury progression, loss of functional cardiomyocytes occurs due to an overlap of several processes including necrosis, apoptosis and inflammation (O'Neal et al. 2012). The present results clearly revealed the dose-dependent cardioprotective effects of TQ in ameliorating ISO-induced myocardial injury. While the cardiotoxic effects of ISO were attenuated in rats pretreated with the low and high doses of TQ, the high dose provided better cardioprotection than the low dose.

Recently, herbal medicine is increasingly gaining greater acceptance from the public and medical profession due to greater advances in understanding mechanisms by which herbal agents positively influence health and quality of life (Arbeláez et al. 2018; Caricati-Neto et al. 2019). Thymoquinone is the basic pharmacologically active component of the essential oil obtained from the extract of black cumin (Nigella sativa) seeds (Ali and Blunden 2003). It has been shown to have antioxidant and antiinflammatory properties with multiple therapeutic benefits especially in cardiovascular diseases (Shabana et al. 2013). Also, previous studies in rats have shown that TQ exerts a cardioprotective effect against myocardial ischemic injury and infarction (Ojha et al. 2015; Hassan et al. 2017). However, the detailed mechanisms of TQ cardioprotection have not been fully clarified and, to the best of our knowledge, the effect of TQ dose on its cardioprotective against myocardial injury has not been tested. These issues were considered in the present study using the ISO-induced myocardial injury model in rats.

In our study, the induction of myocardial injury was associated with a significant increase in serum levels of AST, CK-MB, LDH and troponin I in all ISO-administered rats compared to control rats. These results were indicative of the severity of myocardial injury and confirmed by the cardiac histopathological changes and highly significant correlations between the levels of serum biomarkers and measures of the infarct area. Thymoquinone pretreatment produced a significant reduction in the infarct area, the current gold standard of cardioprotection in experimental studies (Heusch et al. 2019). It also significantly attenuated the elevation in the levels of serum cardiac biomarkers in ISO-treated rats. Additionally, the present study demonstrated that the extent of improvement in serum biomarkers was dose-dependent and normalization of serum CK-MB activity and troponin I level was observed in rats treated with the larger dose of TQ.

With the development of myocardial injury, excessive production of ROS occurs causing a disturbance in the physiological balance between free radicals and the endogenous antioxidant defense system $(\mathrm{He}$ and Zuo 2015). Furthermore, it has been documented that the heart is highly susceptible to oxidative stress, compared to other organs, due to the lower activity of the myocardial antioxidant defense system (Hrelia et al. 2004) In the present study, control rats treated with the high dose of TQ showed a significant increase in the baseline GSH myocardial level, compared to control rats. No similar effect was observed in control rats treated with the lower TQ dose. To our knowledge, the present investigation is the first 
reported research work demonstrating this dose-dependent effect of TQ on the basal antioxidant state of the myocardium, an effect that could be attributed to the increase in the expression of antioxidant genes following TQ treatment (Ismail et al. 2010).

In ISO-administered rats, in this study, the myocardial MDA level increased with a concomitant decrease in the two antioxidant markers (GSH and SOD). The increase in the myocardial MDA, the main lipid peroxidation product, might have resulted due to excessive ROS production by auto-oxidation of ISO (Wang et al. 2016).. Also, our results demonstrated that, in rats pretreated with TQ before ISO administration, the myocardial MDA level decreased and GSH content and SOD activity increased, compared to the ISO group. Additionally, this ameliorative effect of TQ was more efficacious with the use of the high dose, compared to the low dose. This observation was also supported by the findings that serum cardiac biomarkers and infarct area measures correlated positively with myocardial MDA levels and negatively with the myocardial levels of antioxidants (GSH and SOD).

Typically after myocardial injury induction, an inflammatory response which is closely linked with oxidative stress occurs in the heart ( Kruk et al. 2019). This response is characterized by increased production and release of the proinflammatory cytokines, IL-1 $\beta$, IL- 6 and TNF- $\alpha$ from the injured cardiomyocytes (Aoyagi and Matsui 2011). These cytokines influence neutrophils recruitment and mediate cellular pathways that result in myocyte hypertrophy, necrosis and apoptosis (de Haan et al. 2013). The results of our study showed a significant increase in myocardial levels of IL-1 $\beta$, IL- 6 and TNF$a$ in ISO- administered rats, compared to control rats. Also, the myocardial levels of these cytokines correlated positively with the serum cardiac biomarkers, myocardial MDA levels and infarct area measures, and negatively with myocardial GSH and SOD levels. These findings were further confirmed by the histopathology as microscopic examination of heart sections from ISO-administered rats showed evidences of myocardial cell death, inflammatory cell infiltration and widespread fibrous tissue deposition in the interstitial and perivascular areas. Rats pretreated with TQ demonstrated reversal of the myonecrosis and inflammation seen in the vehicle-pretreated ISO group with a significant reduction in the myocardial levels of the proinflammatory cytokines. Several mechanisms have been suggested for the TQ anti-inflammatory effect including inhibition of the cyclooxygenase and 5-lipoxidase pathways (Houghton et al. 1995), reduction of gene expression and release of IL-1 $\beta$, IL-6 and TNF-a ( Liu et al. 2019) and suppression of lipid peroxidation in liposomes in addition to its action as a potent free radical scavenger (Badary et al. 2003).

In addition, TQ pretreatment, in the present study, showed a remarkable antifibrotic effect against ISOinduced myocardial fibrosis. Rats treated with TQ before ISO administration exhibited less fibrous tissue deposition in the myocardium as indicated by the histopathological examination of cardiac sections, compared to the vehicle-pretreated ISO group. The mechanism of this effect may be related to the TQinduced downregulation of the expression of the protein levels of transforming growth factor beta, collagen I, collagen III and a- smooth actin that play a crucial role in the pathogenesis of myocardial fibrosis (Bujak and Frangogiannis 2007; Pei et al. 2018). 
The phenomenon of apoptosis has been described as a tissue response to oxidative stress and proinflammatory cytokines and in relation to myocardial infarction (Anversa et al. 1998). In the present study, caspase-3 activity, a reliable marker of apoptosis, was significantly increased in the myocardial tissue of the vehicle-pretreated ISO-administered rats and a significant positive correlation was observed between the levels of caspase-3 activity and infarct area measures. Also, our results revealed that TQ pretreatment in ISO-administered rats significantly inhibited apoptosis as indicated by a significant decrease in myocardial caspase- 3 activity and myocardial infarct area measures. These findings were in agreement with previous studies that have reported a significant decrease of tissue caspase- 3 activity by TQ treatment in rat models of gentamicin-induced liver injury and ischemia/reperfusion-induced injury in isolated rat heart (Galaly et al. 2014; Xiao et al. 2018). Additionally, our results demonstrated that this antiapoptotic effect of TQ was dose-dependent as the myocardial caspase-3 level was significantly reduced in ISO-administered rats pretreated with TQ at both doses ( 20 and $50 \mathrm{mg} / \mathrm{kg} / \mathrm{day}$ ) with a significantly greater reduction with the high dose than with the low dose use.

Accumulating evidence identifies autophagy as the cellular control mechanism of degradation and recycling of dysfunctional cellular components (Wang et al. 2018). In the heart, although autophagy can be beneficial, unregulated and sustained autophagy can be deleterious and inhibition of autophagy can lead to adverse cardiac remodeling following MI (Tommasino et al. 2015). Thus, one of the approaches in cardioprotection is to suppress excessive autophagy without eliminating its basal level (Xie et al. 2011). In the present study, rat treatment with the high dose of TQ resulted in a significant increase in myocardial autophagy as indicated by the increased basal level of LC3, a marker of autophagy (Mizushima \& Yoshimori 2007). On the contrary, ISO administration to the rats caused a significant decline in the myocardial LC3 level as compared to control rats indicating inhibition of autophagy in cardiomyocytes. These results were in confirmation with earlier studies which have reported reduced autophagy in cardiac damage caused by other agents or pathological conditions (Zheng et al. 2017; Zilinyi et al. 2018; Liu et al. 2019). Also, our study showed that TQ pretreatment in ISO-administered rats resulted in a significant dose-dependent increase in myocardial LC3 level, compared to vehicle-pretreated ISO-administered rats. These results are indicative of improved autophagy in the hearts of ISO-

administered rats pretreated with TQ. This autophagy-enhancing effect provided additional benefits to TQ cardioprotection as indicated by the highly significant inverse correlation between myocardial tissue LC3 levels and infarct area measures in rats with induced myocardial injury.

\section{Conclusion}

This study demonstrated that TQ pretreatment exhibited a dose-dependent cardioprotective effect against ISO-induced myocardial injury through integrated mechanisms depending on its antioxidant, antiinflammatory, antiapoptotic, antifibrotic and autophagy enhancing effects. These findings give several new and important insights into the efficacy of TQ in ameliorating the myocardial injury-associated changes in serum biomarkers and myocardial biochemical and histological features. The clinical implications of the present study remain to be elucidated with further investigation to raise the possibility 
that TQ may serve as a promising prophylactic therapy for patients who are at risk of developing $\mathrm{MI}$ and to provide cardioprotection against the progression of existent infarction in diagnosed cases.

\section{Supporting Information}

- - Supplementary material I: Excel files showing original data

- - Supplementary material II:

Myocardial infarction area measurement

Determination of microtubule-associated protein light chain 3 (LC3) level

\section{Declarations}

\section{Ethics Approval}

The protocol of this study was approved by the Animal Care and Use Committee of the Medical Research Institute, Alexandria University (Alexandria, Egypt). All experimental procedures related to the rats were carried out according to the instructions of the European Directive 2010/63/EU for animal experimentation and the guidelines of the Animal Care and Use Committee, Medical Research Institute, Alexandria University.

Consent to Participate: Not applicable

Consent to Publish: Not applicable

\section{Authors Contributions}

M.M.F. conceived the idea of this work and made its design. A.A.K. and W.F.E. conducted the experimental work. R.M.R. carried out the histopathological work. M.M.F. analyzed and interpreted the data, and wrote the article. All authors read and approved the article before submission. The authors declare that all data were generated in-house and that no paper mill was used.

Funding sources: No grants or funds were received in support of this study.

Competing Interests: The authors declare that they have no conflicts of interest regarding this study.

\section{References}

Ahmad A, Husain A, Mujeeb M, Khan SA, Najmi AK, Siddique NA (2013) A review on therapeutic potential of Nigella sativa: A miracle herb. Asian Pac J Trop Biomed 3 (5): $337-352$ 
Ali BH, Blunden G (2003) Pharmacological and toxicological properties of Nigella sativa.

Phytother Res 17(4): 299-305

Anversa P, Cheng W, Liu Y, Leri A, Redaelli G, Kajstura J (1998) Apoptosis and

myocardial infarction. Basic Res Cardiol 93 (Suppl. 3): 8-12

Aoyagi T, Matsui T (2011) The cardiomyocyte as a source of cytokines in cardiac injury.

J Cell Sci Ther 2012: 1-6

Arbeláez LF, Pardo AC, Fantinelli JC, Schinella GR, Mosca SM, Ríos J-L (2018)

Cardioprotection and natural polyphenols: an update of clinical and experimental studies.

Food Funct 9: 6129-6145.

Awad AS, Abd Al-Haleem EN, El-Bakly WM, Sherief MA (2016) Thymoquinone

alleviates nonalcoholic fatty liver disease in rats via suppression of oxidative stress, inflammation, apoptosis. Naunyn-Schmiedeberg $\otimes \mathrm{s}$ Arch Pharmacol 389(4): 381-391

Badary OA, Taha RA, Gamal el-Din AM, Abdel-Wahab MH (2003) Thymoquinone is

a potent superoxide anion scavenger. Drug Chem Toxicol 26 (2): 87-98

Bujak M, Frangogiannis NG (2007) The role of TGF-beta signaling in myocardial infarction and cardiac remodeling. Cardiovasc Res 74(2): 184-195

Caricati-Neto A, Errante PR, Menezes-Rodrigues FS (2019) Recent advances in pharmacological and non-pharmacological strategies of cardioprotection. Int J Mol Sci 20 (16): 4002

de Haan JJ, Smeets MB, Pasterkamp G, Arslan F (2013) Danger signals in the initiation of the inflammatory response after myocardial infarction. Mediat Inflamm 2013: 206039

Farag MM, Ahmed GO, Shehata RR, Kazem AH (2015) Thymoquinone improves the kidney and liver changes induced by chronic cyclosporine A treatment and acute renal ischaemia/reperfusion in rats. J Pharm Pharmacol 67 (5): 731-739 
Galaly SR, Ahmed OM, Mahmoud AM (2014) Thymoquinone and curcumin prevent

gentamicin-induced liver injury by attenuating oxidative stress, inflammation and apoptosis.

J Physiol Pharmacol 65 (6): 823- 832

Gerczuk PZ, Kloner RA (2012) An update on cardioprotection: a review of the latest adjunctive therapies to limit myocardial infarction size in clinical trials. J Am Coll Cardiol 59 (11): 969-978

Halliwell B (1991) Reactive oxygen species in living systems: source, biochemistry, and role in human disease. Am J Med 91: 14s-22s

Hassan Q, Akhtar M, Ahmed S, Ahmad A, Najmi AK (2017) Nigella sativa protects against isoproterenol-induced myocardial infarction by alleviating oxidative stress, biochemical alterations and histological damage. Asian Pac J Trop Biomed 7 (4): 294-299

He F, Zuo L (2015) Redox roles of reactive oxygen species in cardiovascular diseases. Int J Med Sci 16 (11): 27770-27780

Heusch G, Kleinbongard P, Rassaf T (2019) Cardioprotection beyond infarct size reduction.

Circ Res 124: 679-680

Houghton PJ, Zarka R, de las Heras B, Hoult JR (1995) Fixed oil of Nigella sativa and derived thymoquinone inhibit eicosanoid generation in leukocytes and membrane lipid peroxidation. Planta Med 61(1): 33-36

Hrelia S, Bordoni A, Angeloni C, Leoncini E, Biagi P (2004) Nutritional interventions to counteract oxidative stress in cardiac cells. Ital J Biochem 53 (4): 157-163

Ismail M, Al-Naqeep G, Chan KW (2010) Nigella sativa thymoquinone-rich fraction greatly improves plasma antioxidant capacity and expression of antioxidant genes in hypercholesterolemic rats. Free Radic Biol Med 48 (5): 664-672

Klionsky DJ, Cucrvo AM, Seglen PO (2007) Methods for monitoring autophagy from 
yeast to human. Autophagy 3: 181-206

Kruk J, Aboul-Enein HY, Kładna A, Bowser JE (2019) Oxidative stress in biological

systems and its relation with pathophysiological functions: the effect of physical activity on cellular redox homeostasis. Free Radic Res 53(5): 497-521

Leone A (2017) Myocardial infarction: pathological relevance and relationship with coronary risk factors. Curr Pharm Des 23: 3205

Lie JT, Pairolero PC, Holley KE, Titus JL (1975) Macroscopic enzyme-mapping verification of large, homogeneous, experimental myocardial infarcts of predictable size and location in dogs. J Thorac Cardiovasc Surg 69 (4): 599-605

Liu H, Sun Y, Zhang Y, Yang G, Guo L, Zhao Y, et al. (2019) Role of thymoquinone in cardiac damage caused by sepsis from BALB/c mice. Inflammation 42 (2): 516-525

Milei J, Nunez RG, Rapaport M (1978) Pathogenesis of isoproterenol-induced myocardial lesions: its reation to human coagulative myocytolysis. Cardiology 63 (3): 139-151

Mizushima N, Yoshimori T (2007) How to interpret LC3 immunoblotting. Autophagy 3 (6): $542-545$

Moran AE, Forouzanfar MH, Roth GA, Mensah GA, Ezzati M, Murray CJL, et al.

(2014) Temporal trends in ischemic heart disease mortality in 21 world regions, 1980 to 2010: the Global Burden of Disease 2010 Study. Circulation 129 (14): 1483-1492

Nascimento BR, Brant LCC, Marino BCA, Passaglia LG, Ribeiro ALP (2019)

Implementing myocardial infarction systems of care in low/middle-income countries.

Heart 105 (1): 20-26

Nishikimi M, Appaji N, Yagi K (1972) The occurrence of superoxide anion in the reaction of reduced phenazine methosulfate and molecular oxygen. Biochem Biophys Res Commun 46 (2): 849-854 
$0 \otimes$ Neal WT, GriffinWF, Kent SD, Virag JAI (2012) Cellular pathways of death

and survival in acute myocardial. J Clin Exp Cardiol S6: 003

Ohkawa H, Ohishi N, Yagi K (1979) Assay for lipid peroxides in animal tissues by thiobarbituric acid reaction. Anal Biochem 95 (2): 351-358

Ojha S, Azimullah S, Mohanraj R, Sharma C, Yasin J, Arya D S, et al. (2015) Thymoquinone protects against myocardial ischemic injury by mitigating oxidative stress and inflammation. Evid Based Complement Alternat Med 2015: 143629

Ojha S, Goyal S, Kumari S, Arya DS (2012) Pyruvate attenuates cardiac dysfunction and oxidative stress in isoproterenol-induced cardiotoxicity. Exp Toxicol Pathol 64 (4): 393399

Pei Z, Hu J, Bai Q, Liu B, Cheng D, Liu H, et al. (2018) Thymoquinone protects against cardiac damage from doxorubicin-induced heart failure in Sprague-Dawley rats. RSC advances 8 (26): 14633-14639

Richardson RJ, Murphy SD (1975) Effect of glutathione depletion on tissue deposition of methylmercury in rats. Toxicol Appl Pharmacol 31(3): 505-519

Saravanan G, Prakash J (2004) Effect of garlic (Allium sativum) on lipid peroxidation in experimental myocardial infarction in rats. J Ethnopharmacol 94 (1): 155-158

Shabana A, El-Menyar A, Asim M, Al-Azzeh H, Al Thani H (2013) Cardiovascular benefits of black cumin (Nigella sativa). Cardiovasc Toxicol 13 (1): 9-21

Somaiya R, Patel H, Handave M (2015) Potential future therapies of myocardial ischemia reperfusion injury. Indian J Cardio Biol Clin Sci 2: 105-112

Tommasino C, Marconi M, Ciarlo L, Matarrese P, Malorni W (2015) Autophagic flux and autophagosome morphogenesis require the participation of sphingolipids. Apoptosis 20 (5): $645-657$ 
Upaganlawar A, Gandhi H, Balaraman R, (2011) Isoproterenol induced myocardial

infarction: Protective role of natural products. J Pharmacol Toxicol 6 (1): 1-17

Wang X, Guo Z, Ding Z, Mehta JL (2018) Inflammation, autophagy, and apoptosis after myocardial infarction. J Am Heart Associat 7 (9): e008024

Wang Z, Zhang J, Ren T, Dong Z (2016) Targeted metabolomic profiling of cardioprotective effect of Ginkgo biloba L. extract on myocardial ischemia in rats. Phytomedicine 23 (6): 621-631

Xiao J, Ke ZP, Shi Y, Zeng Q, Cao Z (2018) The cardioprotective effect of thymoquinone on ischemia-reperfusion injury in isolated rat heart via regulation of apoptosis and autophagy. J Cell Biochem 119 (9): 7212-7217

Xie M, Morales CR, Lavandero S, Hill JA (2011) Tuning flux: autophagy as a target of heart disease therapy. Curr Opin Cardiol 26 (3): 216-222

Zheng Y, Gu S, Li X, Tan J, Liu S, Jiang Y, et al. (2017) Berbamine postconditioning protects the heart from ischemia/reperfusion injury through modulation of autophagy. Cell Death Dis 8 (2): e2577

Zilinyi R, Czompa A, Czegledi A, Gajtko A, Pituk D, Lekli I, et al. (2018) The cardioprotective effect of metformin in doxorubicin-induced cardiotoxicity: The role of autophagy. Molecules 23 (5): e1184

\section{Tables}

\section{Table 1}

\section{Effects of thymoquinone (TQ) on serum cardiac biomarkers in isoproterenol (ISO) treated rats}




\begin{tabular}{|lllll|}
\hline \multirow{2}{*}{ Experimental groups } & \multicolumn{4}{c|}{ Serum cardiac markers } \\
\cline { 2 - 5 } & $\begin{array}{l}\text { AST } \\
(\mathrm{U} / \mathrm{L})\end{array}$ & CK-MB & LDH & c Tn I \\
& $(\mathrm{U} / \mathrm{L})$ & $(\mathrm{pg} / \mathrm{ml})$ & $(\mathrm{ng} / \mathrm{ml})$ \\
\hline Control & $29.00 \pm 1.04$ & $5.48 \pm 0.23$ & $46.93 \pm 2.94$ & $0.40 \pm 0.01$ \\
TQ20 & $26.88 \pm 0.44$ & $3.49 \pm 0.13$ & $37.14 \pm 0.92$ & $0.31 \pm 0.01$ \\
TQ50 & $23.13 \pm 0.55$ & $3.38 \pm 0.12$ & $30.63 \pm 0.84$ & $0.23 \pm 0.01$ \\
ISO & $118.10 \pm 3.70^{\mathrm{a}}$ & $30.44 \pm 1.57^{\mathrm{a}}$ & $260.30 \pm 16.17^{\mathrm{a}}$ & $3.65 \pm 0.30^{\mathrm{a}}$ \\
TQ20+ISO & $54.71 \pm 1.80^{\mathrm{ab}}$ & $11.49 \pm 0.36^{\mathrm{ab}}$ & $114.60 \pm 3.29^{\mathrm{ab}}$ & $1.43 \pm 0.04^{\mathrm{ab}}$ \\
TQ50+ISO & $40.50 \pm 1.26^{\mathrm{abc}}$ & $7.80 \pm 0.36^{\mathrm{bc}}$ & $81.28 \pm 3.65^{\mathrm{abc}}$ & $0.82 \pm 0.05^{\mathrm{bc}}$ \\
\hline
\end{tabular}

Values shown are mean \pm SEM $(n=6-8$ rats /group).

$\mathbf{a}_{\mathrm{P}}<0.05$ versus control group.
$\mathbf{b}_{\mathrm{P}}<0.05$ versus ISO group.
$\mathbf{c}_{\mathrm{P}}<0.05$ versus TQ $20+$ ISO group.

Table 2

Correlations between serum cardiac markers and cardiac tissue parameters

\begin{tabular}{|c|c|c|c|c|}
\hline \multirow{2}{*}{ Cardiac tissue parameters } & \multicolumn{4}{|c|}{ Serum cardiac markers } \\
\hline & AST & CK-MB & LDH & Troponin-I \\
\hline \multicolumn{5}{|l|}{-Cytokines } \\
\hline IL- $1 \beta$ & $0.977^{a}$ & $0.979^{a}$ & $0.982^{\mathrm{a}}$ & $0.956^{\mathrm{a}}$ \\
\hline IL-6 & $0.992^{\mathrm{a}}$ & $0.987^{a}$ & $0.986^{a}$ & $0.969^{a}$ \\
\hline TNF- $\alpha$ & $0.982^{\mathrm{a}}$ & $0.983^{a}$ & $0.982^{\mathrm{a}}$ & $0.961^{\mathrm{a}}$ \\
\hline \multicolumn{5}{|l|}{$\begin{array}{l}\text { - Oxidative } \\
\text { stress indices }\end{array}$} \\
\hline MDA & $0.988^{a}$ & $0.990^{\mathrm{a}}$ & $0.989^{a}$ & $0.960^{\mathrm{a}}$ \\
\hline GSH & $-0.887^{a}$ & $-0.861^{a}$ & $-0.871^{a}$ & $-0.860^{a}$ \\
\hline SOD & $-0.914^{a}$ & $-0.894^{a}$ & $-0.907^{a}$ & $-0.881^{a}$ \\
\hline
\end{tabular}


AST, aspartate aminotransferase; CK-MB, creatine kinase-myocardial band isoenzyme ; $\mathrm{LDH}$,

lactate dehydrogenase; IL- $1 \beta$, interleukin-1 beta; IL-6, interleukin-6; TNF- $\alpha$, tumor necrosis

factor-alpha; MDA, malondialdehyde ;GSH, reduced glutathione; SOD, superoxide dismutase.

${ }^{\text {a }} \mathrm{P}<0.001(\mathrm{n}=44)$

\section{Figures}

a
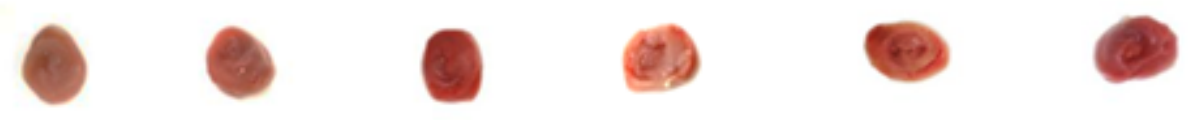

b

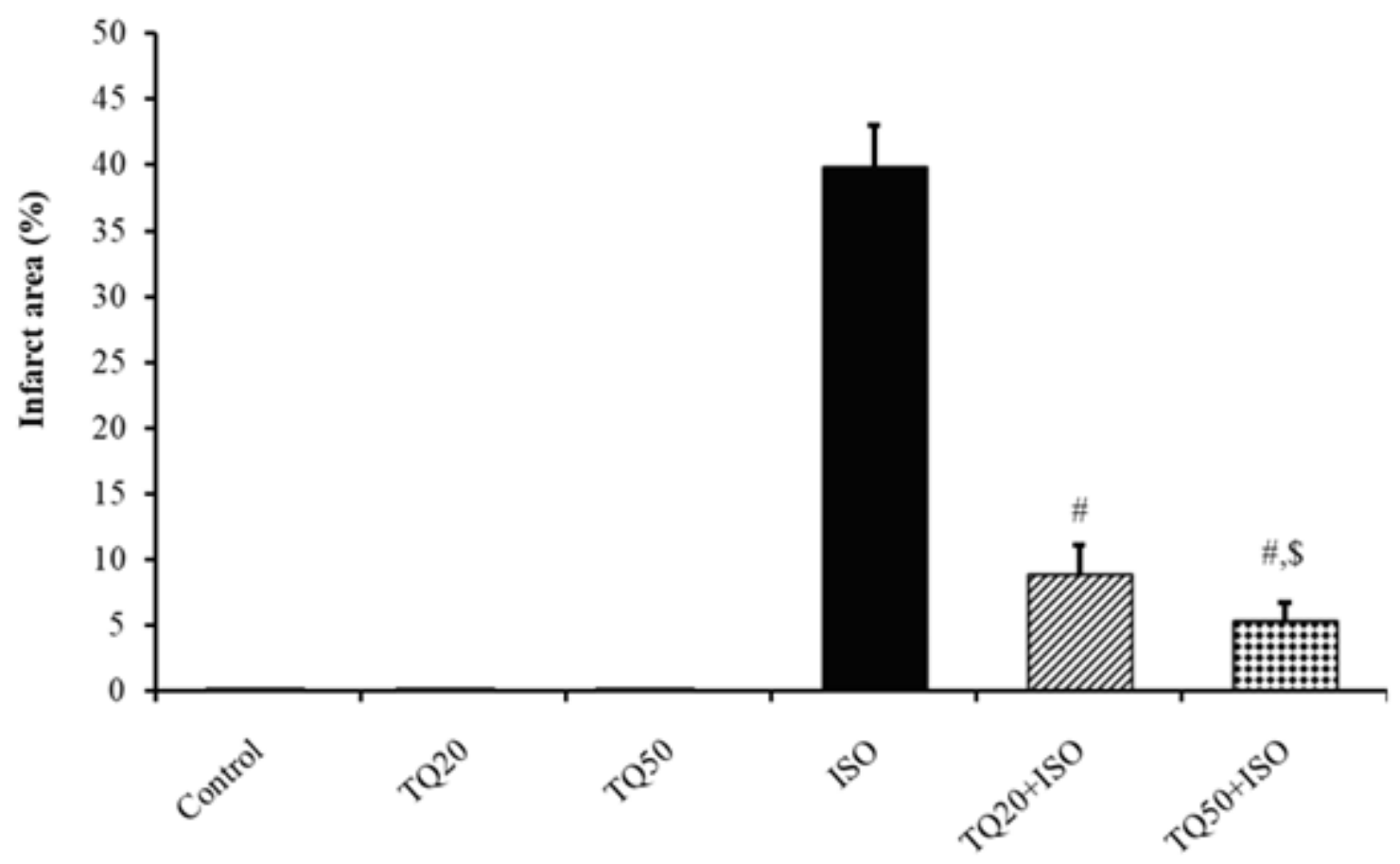

Figure 1

Effect of thymoquinone (TQ) on cardiac tissue infarct area in isoproterenol (ISO) - treated rats using the direct triphenyl tetrazoilium chloride (TTC) stain assay method. a: Images of heart tissues from control, TQ and/or ISO treated rats. b: Effect of TQ on the area of myocardial infarction induced by ISO treatment. Values shown are mean \pm SEM ( $n=6$ rats / group). \# $P<0.05$ as compared to the ISO group. $\$ P<0.05$ as compared to the TQ20 + ISO group 


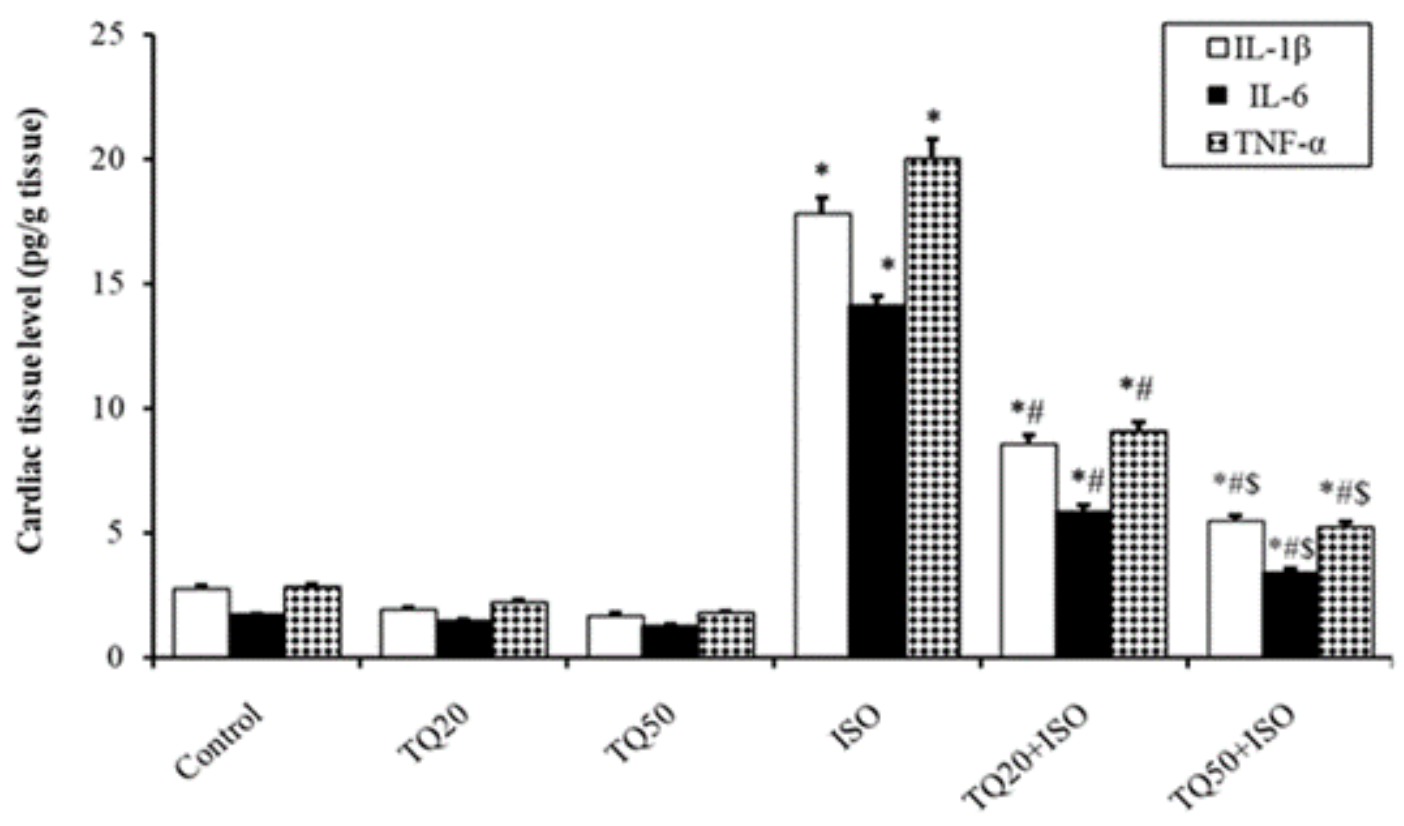

Figure 2

Effects of thymoquinone (TQ) on cardiac tissue interleukin (IL)-1 $1 \beta$, IL-6 and tumor necrosis factor -alpha (TNF-a) levels in isoproterenol (ISO) -treated rats. Values shown are mean \pm SEM ( $n=6-8$ rats/ group). * $P$ $<0.05$ as compared to the control group. \# $\mathrm{P}<0.05$ as compared to the ISO group. $\$ \mathrm{P}<0.05$ as compared to TQ20 + ISO group.

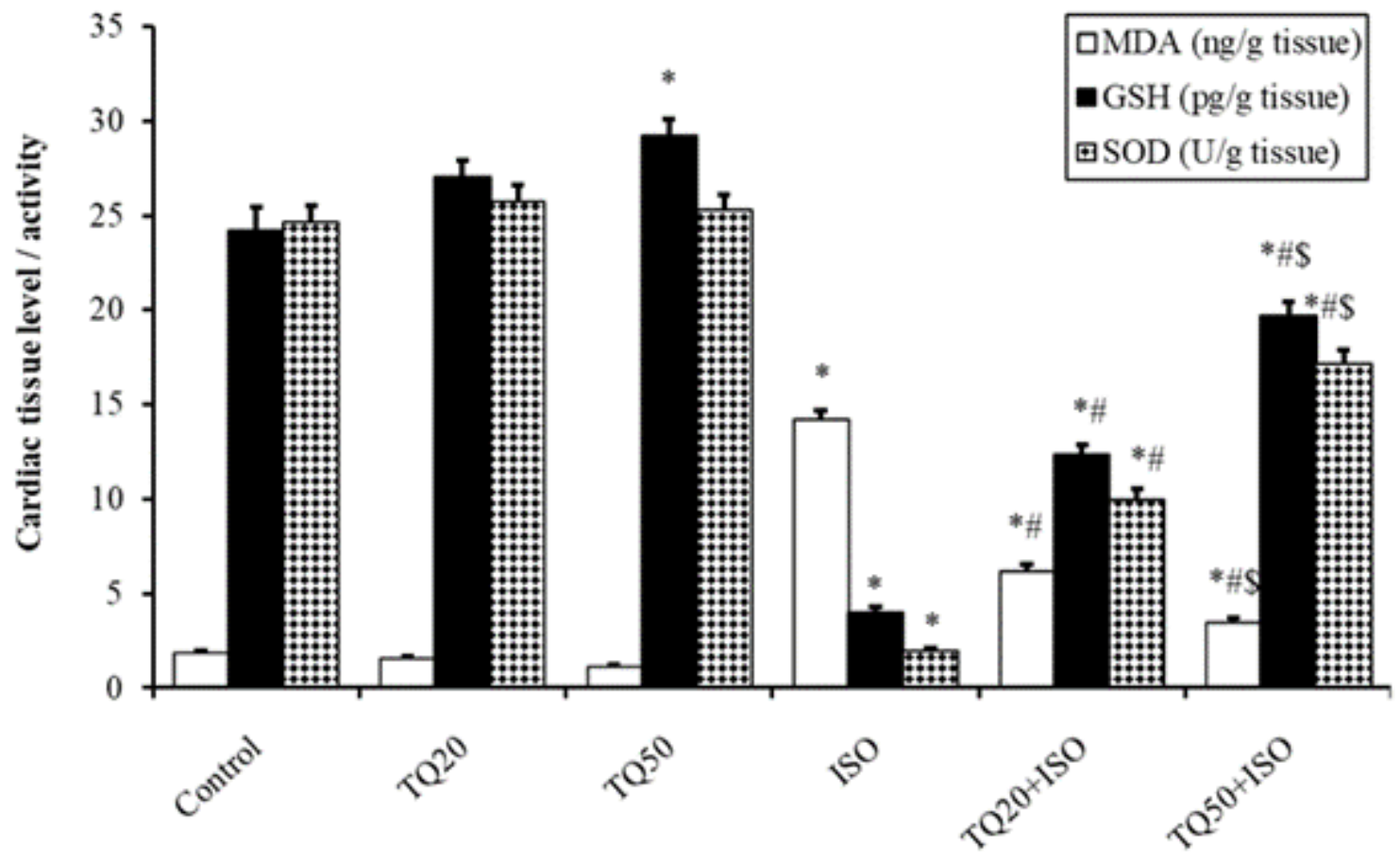

Figure 3 
Effects of thymoquinone (TQ) on cardiac tissue malondialdehyde (MDA) and reduced glutathione (GSH) contents and superoxide dismutase (SOD) activity in isoproterenol (ISO) - treated rats. Values shown are mean \pm SEM ( $n=6-8$ rats/group). ${ }^{*} P<0.05$ as compared to the control group. \# $P<0.05$ as compared to the ISO group. $\$ \mathrm{P}<0.05$ as compared to TQ20 + ISO group

a

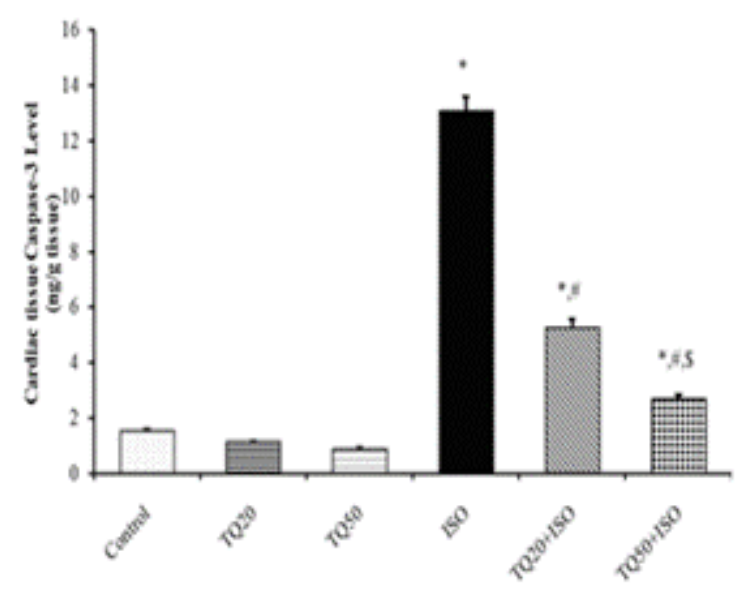

b

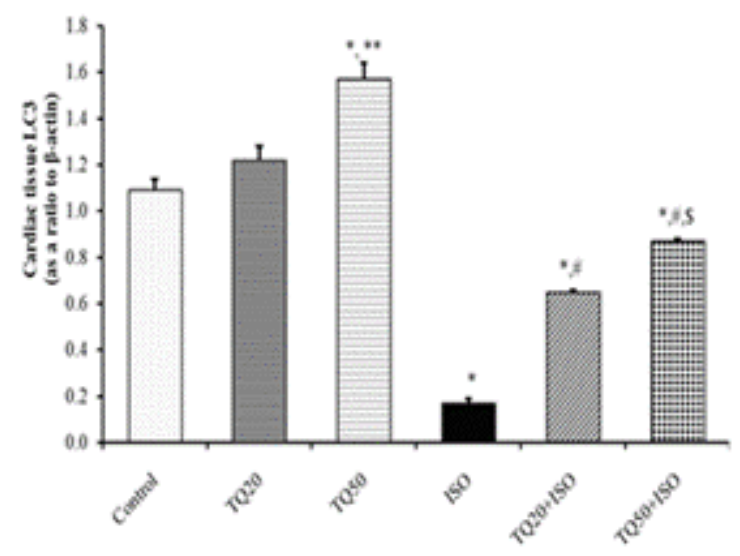

c

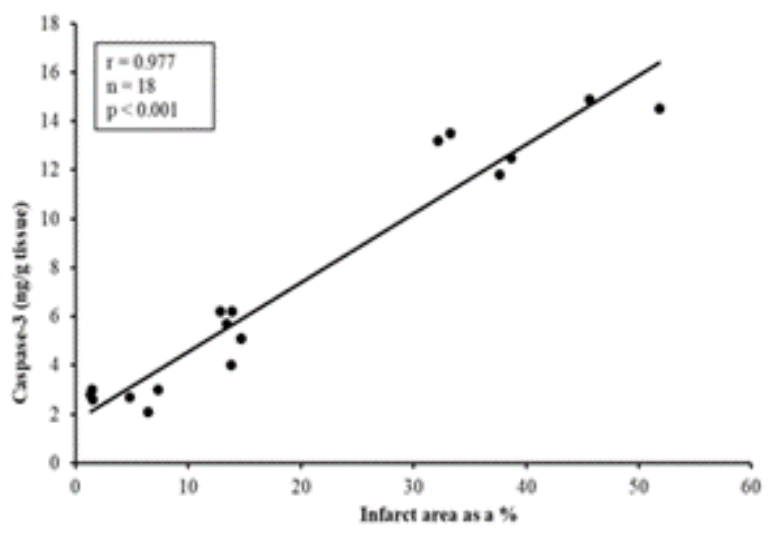

d

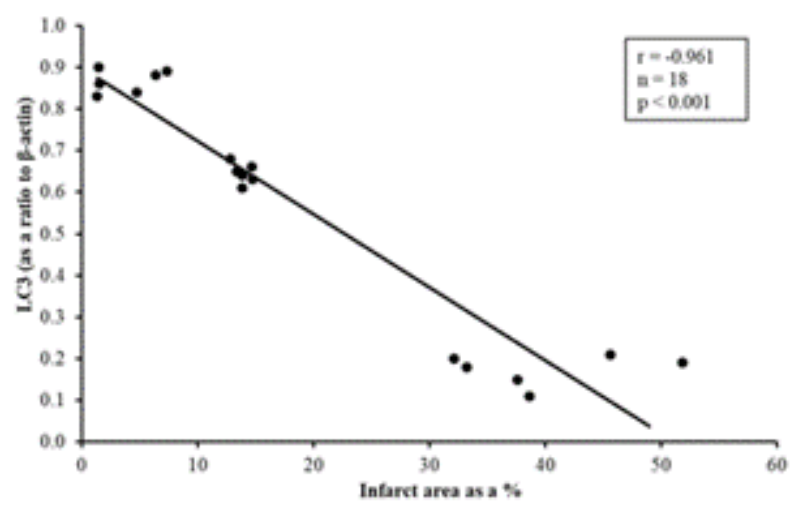

Figure 4

Effect of thymoquinone (TQ) on cardiac tissue caspase -3 level (a) and microtubule- associated protein light chain 3 (LC3) level (b) in isoproterenol (ISO)-treated rats. Values shown are mean \pm SEM ( $n=6-8$ rats/group). ${ }^{*} \mathrm{P}<0.05$ as compared to the control group. ${ }^{*} \mathrm{P}<0.05$ as compared to the TQ20 group. \# $\mathrm{P}$ $<0.05$ as compared to the ISO group. $\$ P<0.05$ as compared to TQ20 + ISO group. Figs. $c \& d$ show the correlations between the myocardial infract area measures and the cardiac tissue levels of both caspase3 (c) and microtubule-associated protein light chain3 (d) 
a

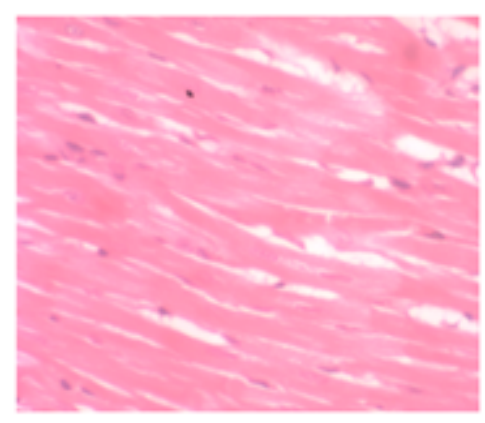

d

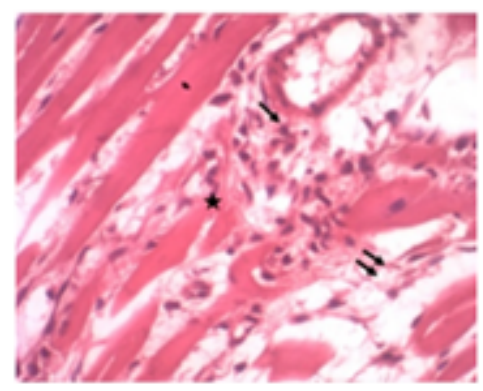

b

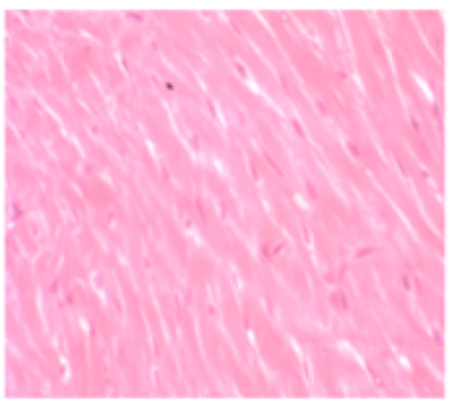

c

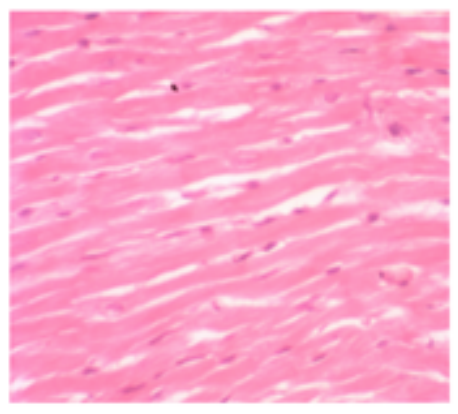

f
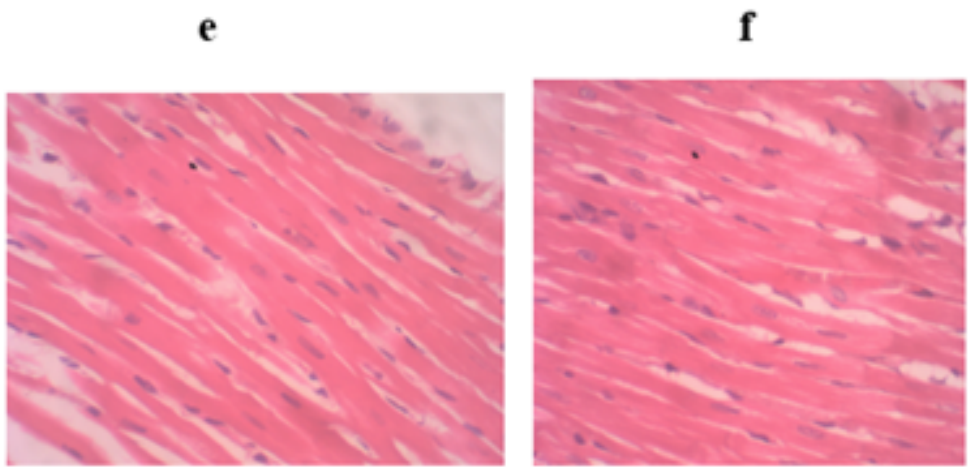

Figure 5

See manuscript for full figure caption. 


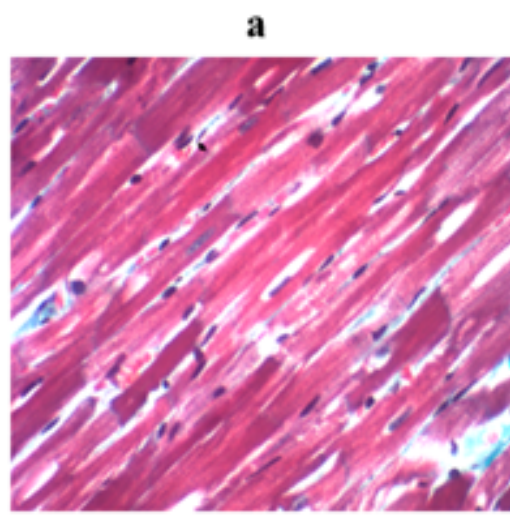

b
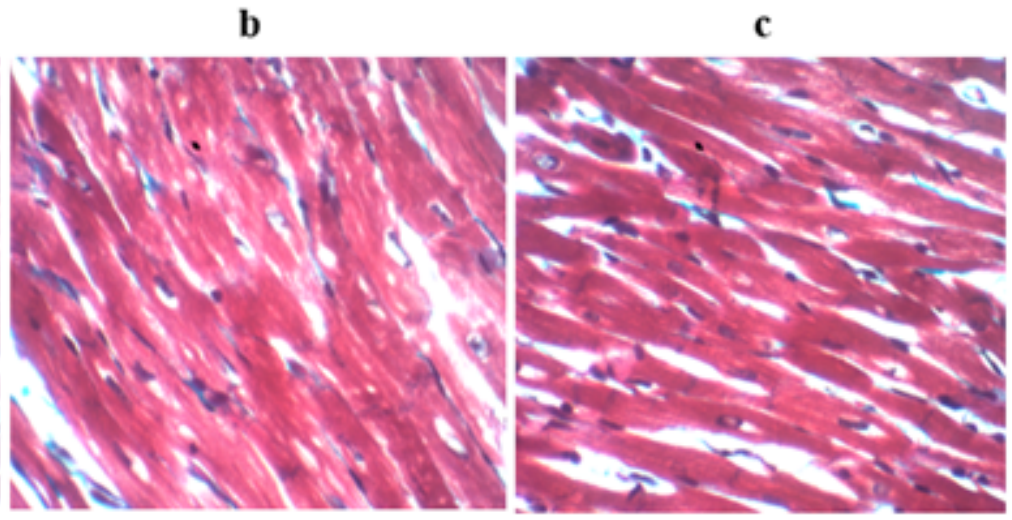

d

e

f

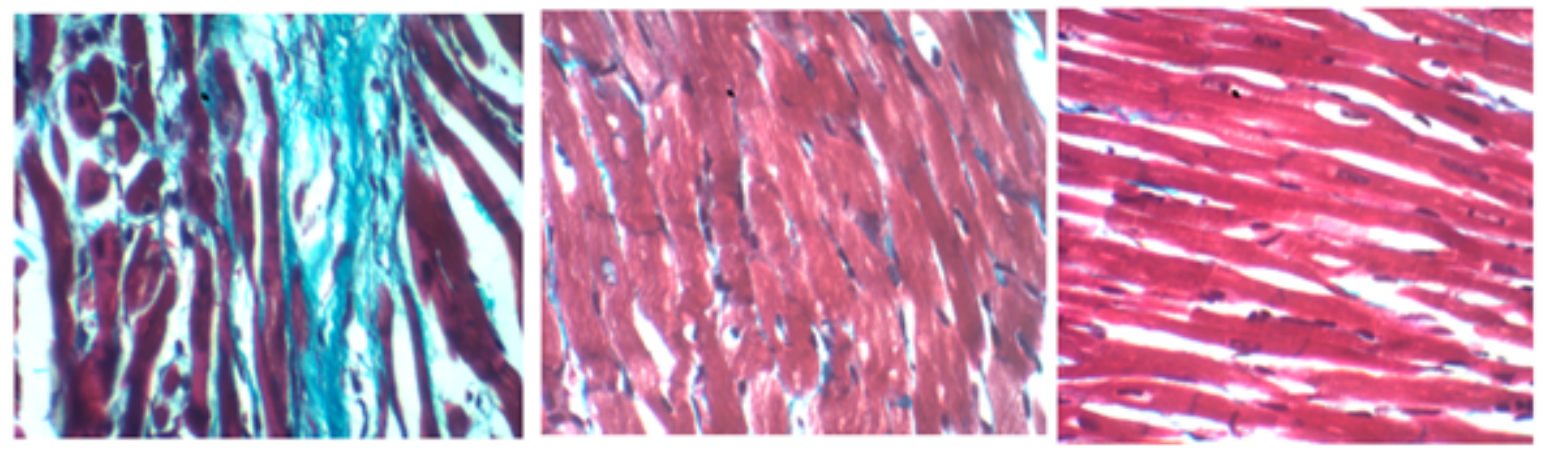

Figure 6

Photomicrographs of cardiac tissue sections from control rats treated with the vehicle (Fig. a) or thymoquinone at doses of 20 and $50 \mathrm{mg} / \mathrm{kg} /$ day (Figs. b and c, respectively) showing scanty interstitial delicate collagen fibrils stained in blue in association with normal cardiac histoarchitecture. (d): A photomicrograph of a cardiac tissue section from ISO-treated rats showing thick dense amounts of collagen fibrils stained in blue in association with necrotic areas of the cardiac tissue. (e and $f$ ): Photomicrographs of cardiac tissue sections from the TQ20+ISO and TQ50+ISO groups, respectively, showing few interstitial collagen fibrils with marked resemblance to normal cardiac histoarchitecture (Masson's trichrome stain, $\mathrm{x} 400$ ).

\section{Supplementary Files}

This is a list of supplementary files associated with this preprint. Click to download.

- LC3markersimagesstatistics.docx

- collectivesheetsoforiginaldata.xlsx 